\title{
Kernohan-Woltman Notch Phenomenon Caused by a Traumatic Epidural Hematoma
}

\author{
Jin-Hyung Lee ${ }^{\star}$ (D), Mi-Ri Kang ${ }^{\dagger}$ (D), Sang Jin $\mathrm{Kim}^{\dagger}$ (D), Bong-Goo Yoo* (D), Eung Gyu Kim ${ }^{\dagger}$ (D), Ki-Hwan ji ${ }^{\dagger}$ (D) \\ Department of Neurology, Kosin University Gospel Hospital ${ }^{\star}$, Busan; Department of Neurology, Busan Paik Hospital, Inje University College of \\ Medicine ${ }^{\dagger}$, Busan, Korea
}

The Kernohan-Woltman notch phenomenon is one of the false localizing signs and hinders physicians' accurate judgment of lesion localization. The Kernohan-Woltman notch phenomenon has been rarely reported in patients with acute cerebral hemorrhage, especially acute or subacute traumatic subdural hemorrhage. The mechanism is a transtentorial herniation squeezing the opposite side of the cerebral crus against the tentorial edge of the cerebellum. If the brain image and neurological examination are inconsistent in a patient with traumatic brain injury, the Kernohan-Woltman notch phenomenon should be in the list of differential diagnoses. Herein, we present the case of a 25-year-old woman with epilepsy who presented with ipsilateral limb weakness caused by an acute traumatic epidural hematoma associated with seizure attack.

\section{J Neurosonol Neuroimag 2019;11(2):1\$2-1\$5}

Key Words: Traumatic brain injury; Hemiparesis; Epidural hematoma; Diagnostic errors; Neurologic examination
Received: November 11, 2019

Revised: December 3, 2019

Accepted: December 5, 2019

Address for correspondence: Ki-Hwan Ji

Department of Neurology, Busan Paik Hospital, Inje University College of Medicine, 75 Bokji-ro, Busanjin-gu, Busan 47392, Korea

Tel: $+82-51-890-8613$

Fax: +82-51-890-6130

E-mail:kihwanji@gmail.com
Despite the availability of various imaging tools such as computed tomography (CT), magnetic resonance imaging (MRI), and diffusion tensor tractography in the modern era, it is neurological signs that often provide clear information of the lesion location and may provide evidence of pathological characteristics. However, physicians may sometimes encounter obstacles such as false localizing signs that lead to failure to infer the proper lesion lateralization and localization based on the neurological signs. The Kernohan-Woltman notch phenomenon is one of the false localizing signs and manifests as an ipsilateral limb weakness associated with supratentorial lesions such as those caused by increased intracranial pressure. ${ }^{1}$ Herein, we present a case of Kernohan-Woltman notch phenomenon in a patient who showed limb weakness on the same side as an acute traumatic epidural hematoma.

\section{CASE REPORT}

A 25-year-old woman with epilepsy presented to the emergency department (ED) because of decreased mental status. Absence seizures (eye blinking and upward rolling of the eyes) and subtle myoclonic seizures were first observed when she was 12 years of age, and generalized tonic-clonic seizures started at the age of 14 years. She had been taking lamotrigine $200 \mathrm{mg}$ daily, and seizures were rare for $>9$ years. However, the frequency of absence seizures increased during pregnancy, occasionally requiring hospitalization, and the lamotrigine dose was increased to $300 \mathrm{mg}$ per day. Three days before the present admission, she gave birth to a healthy baby boy at 38 weeks of gestation by natural delivery. On the day of admission, she lost consciousness while on the phone and was found lying next to her bed. An unwitnessed epileptic seizure was suspected. She suffered trauma to her right head, which appeared to have resulted from falling and bumping her head. She 
was deeply drowsy and had frequent absence seizure attacks during the ED admission. On neurological examination, she had no muscle weakness, and her pupils were equal in size and constricted equally in response to light stimulus. Owing to her drowsy mental state, the extraocular movement could not be adequately checked, but no eyeball deviation was observed. Brain CT revealed an acute right temporoparietal epidural hematoma with a midline shift (Fig. 1A, B). She underwent an urgent decompressive craniotomy and hematoma removal. She was admitted to the intensive care unit, where she received supportive care. As her mental state improved, we noticed right-limb weakness (grade
3/5). As Todd's paralysis was possible and the follow-up brain CT revealed no acute symptomatic lesion, we carefully observed her neurological changes, but she showed no motor improvement. We reviewed her previous initial brain CT scan and realized that a transtentorial herniation was compressing the left cerebral peduncle. The Kernohan-Woltman notch phenomenon was suspected. On the gth day after admission, follow-up brain MRI revealed a hyperintense lesion in the cerebral crus of the left cerebral peduncle (Fig. 1C-E). One year after, she showed a persistent minor weakness of her right distal arm.
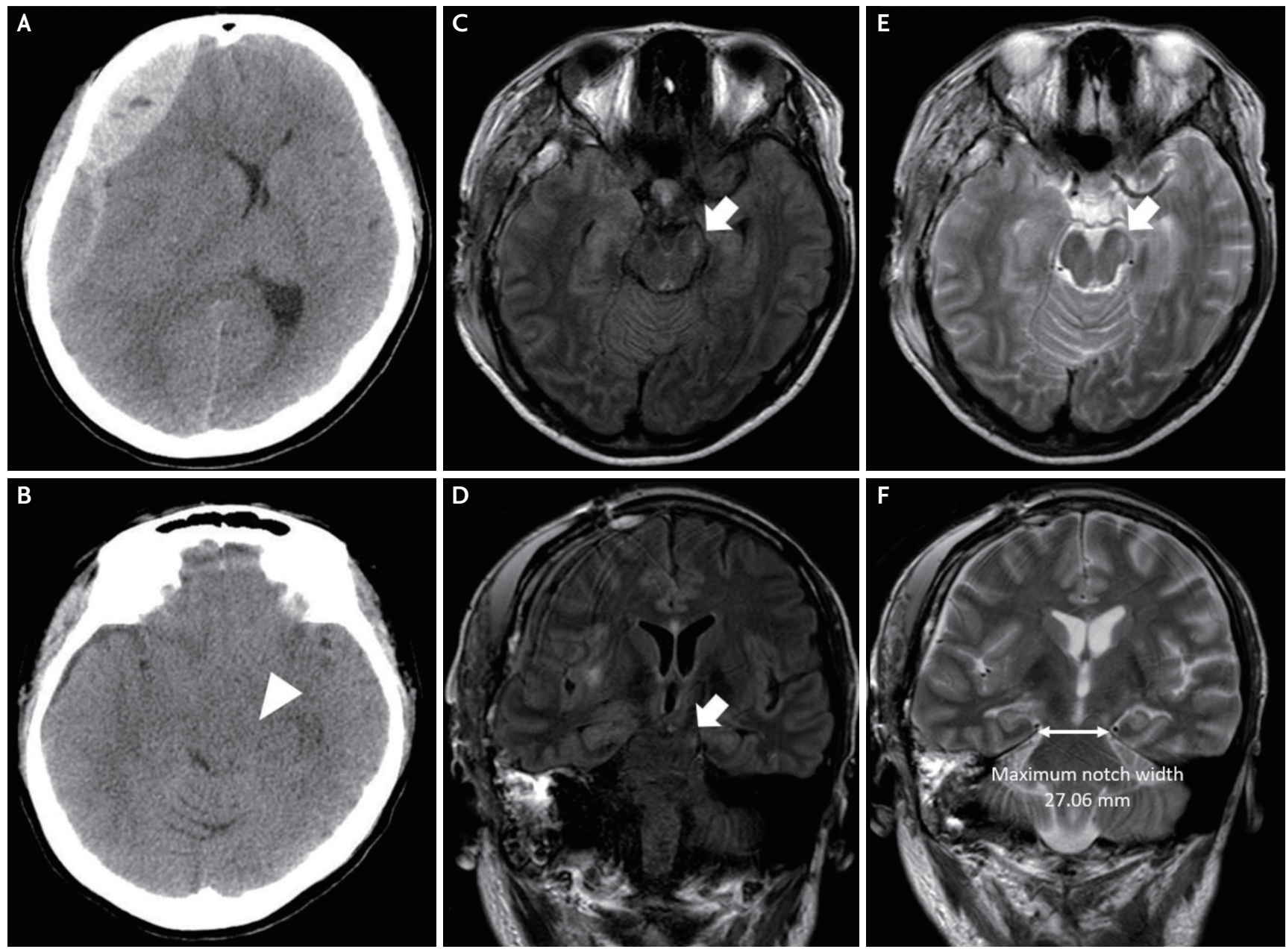

FIG. 1. (A, B) Initial brain computed tomography (CT) and (C, D) follow-up fluid-attenuated inversion recovery axial/coronal and (E, F) T2-weighted axial/coronal magnetic resonance images. (A) The brain CT image shows an epidural hematoma in the right frontotemporal lobe with subfalcine herniation. (B) The transtentorial herniation to the left caused compression of the cerebral peduncle (arrowhead). (C-E) The brain magnetic resonance image obtained 9 days after the injury shows a hyperintense lesion in the left cerebral peduncle (short arrows). (F) The maximum notch width was measured on the coronal image. 


\section{DISCUSSION}

Ipsilateral pupil dilation, contralateral hemiparesis, and decerebrate posture are typical pictures of transtentorial herniation associated with a supratentorial hemispheric lesion. ${ }^{1}$ The mechanism is a downward displacement of the ipsilateral medial temporal lobe into the tentorial hiatus. Around 90 years ago, Kernohan and Woltman ${ }^{2}$ observed a few patients with a cerebral mass who had limb weakness on the same side as the cerebral lesion. Through a postmortem examination, they proposed the mechanism that the descending pressure caused the squeezing of the opposite side of the cerebral crus against the tentorial edge of the cerebellum. ${ }^{2}$ Findings from modern imaging techniques now support their proposal. ${ }^{3-5}$

Adler and Milhorat ${ }^{6}$ reported that the maximum notch width in the axial plane was $29.6 \pm 3.0 \mathrm{~mm}$ in 100 autopsy cases and classified them into a narrow type $(24.5-27 \mathrm{~mm})$ and wide type (32-39 mm). Carrasco et al. proposed the narrow width of tentorial notches as a predisposing factor of the Kernohan-Woltman notch phenomenon in a patient with acute traumatic subdural hematoma. The maximum notch width was 27.06 $\mathrm{mm}$ in our patient (Fig. $1 \mathrm{~F}$ ). While structural variations such as the small width of the tentorial notch may be a possible explanation, the mechanism of transtentorial herniation due to a supratentorial hemispheric mass resulting in different outcomes, that is, contralateral weakness through compression of the ipsilateral medial temporal lobe or ipsilateral weakness through compression of the contralateral cerebral crus, is unclear.

The Kernohan-Woltman notch phenomenon is mainly caused by intracranial hemorrhage, especially acute or chronic subdural hematoma. ${ }^{1,7} \mathrm{~A}$ few studies have reported the association of the Kernohan-Woltman notch phenomenon with epidural hematoma. ${ }^{8}$

Evaluation with brain CT would be sufficient in most patients with a severe traumatic head injury, and the decision for surgical intervention is usually made on the basis of the neurological condition of the patient and brain CT result. Most MRI studies are performed after the surgical intervention., ${ }^{5,7}$ The literature and modern technology indicate that brain MRI is useful for the diagnosis of the Kernohan-Woltman notch phenomenon in patients whose brain CT findings could not explain the focal neurological signs. ${ }^{1,5,7}$

Our case has two points of peculiarity. Initially, the patient presented a deeply drowsy mentality and ongoing seizures, without evident lateralizing signs in the neurological examination. We noticed her rightside weakness when her mentality improved after seizure control, and craniotomy and hematoma removal. Most studies reported and discussed the discordance between the brain image of the mass lesion and the neurological sign, which is the side of the limb weakness, as the initial enigma encountered in patients with intracranial hemorrhage. ${ }^{1}$ A previous report described a case of the Kernohan-Woltman notch phenomenon that led to surgical intervention on the opposite side of the lesion. ${ }^{9}$ In our case, whether the limb weakness developed before or after the hematoma removal is unclear. A focal neurological change is not easy to detect under a sedated state after brain surgery. Therefore, a thorough neurological examination should be warranted before and after the surgical intervention in patients with traumatic intracranial hemorrhage.

In conclusion, the discordance between the brain image and the focal neurological signs in patients with an intracranial mass lesion may require further brain MRI examination, considering the possibility of the Kernohan-Woltman notch phenomenon. As it might not be evident initially or might develop later after surgery, a careful neurological examination is critical even in the modern technology era.

\section{Conflicts of Interest}

No potential conflicts of interest relevant to this article was reported.

\section{REFERENCES}

1. McKenna C, Fellus J, Barrett AM. False localizing signs in traumatic brain injury. Brain Inj. 2009;23:597-601.

2. Kernohan JW, Woltman HW. Incisura of the crus due to contralateral brain tumor. Arch NeurPsych. 1929;21:274-287.

3. Gobert F, Baars JH, Ritzenthaler T, Afathi M, Boulogne S, André-Obadia N, et al. Diagnosing Kernohan-Woltman notch phenomenon by somatosensory evoked potentials in intensive care unit. Clin Neurophysiol. 2018;129:254-257.

4. Jang SG, Pyun SB. Diffusion tensor tractography in two 
cases of Kernohan-Woltman notch phenomenon. Ann Rehabil Med. 2013;37:879-885.

5. Mastronardi L, Puzzilli F, Ruggeri A, Guiducci A. Magnetic resonance imaging findings of Kernohan-Woltman notch in acute subdural hematoma. Clin Neurol Neurosurg. 1999;101:122-124.

6. Adler DE, Milhorat TH. The tentorial notch: anatomical variation, morphometric analysis, and classification in 100 human autopsy cases. J Neurosurg. 2002;96:1103-1112.

7. Carrasco R, Pascual JM, Navas M, Martínez-Flórez P, Man-
zanares-Soler R, Sola RG. Kernohan-Woltman notch phenomenon caused by an acute subdural hematoma. J Clin Neurosci. 2009;16:1628-1631.

8. Ozturk S, Ozturk Y, Ocal O. The first case of Kernohan-Woltman notch phenomenon caused by epidural hematoma in a pediatric patient. Pediatr Neurosurg. 2017;52:181184.

9. Wolf RF, ter Weeme CA, Krikke AP. Kernohan's notch and misdiagnosis. Lancet. 1995;345:259-260. 